\title{
Avaliação da Acurácia Diagnóstica da Angiotomografia Coronária de Múltiplos Detectores
}

\author{
Wilson Albino Pimentel Filho ${ }^{1,2,3,4,6}$, Edson Ademir Bocchi ${ }^{3,4}$, Milton Macedo Soares Neto ${ }^{2,5,6}$, \\ Wellington Borges Custódio1, Paulo Cícero Aidar Maiello1, Julio Domingos ${ }^{1}$, Dorival dos Santos Cardos ${ }^{1}$, \\ Thiago Augusto Rubini Miranda', Waigner Bento Pupin Filho', Jorge Roberto Büchler ${ }^{1}$, \\ Stoessel Figueredo de Assis ${ }^{1}$, Egas Armelin'1, Rubens Sirtoli Filho'
}

\section{RESUMO}

Introdução: A angiotomografia coronária de múltiplos detectores (TCMD) vem ganhando confiança como procedimento diagnóstico não-invasivo da doença arterial coronária (DAC). O objetivo deste estudo foi avaliar o desempenho diagnóstico da TCMD e sua influência na modificação das estratégias de revascularização percutânea. Método: O estudo incluiu dois grupos de pacientes: um grupo principal (GP), composto por 100 pacientes triados com suspeita clínica de DAC grave pela TCMD e indicação de cineangiocoronariografia (CINE), e um grupo controle (GC), para comparação, composto por 100 pacientes selecionados no mesmo período e com indicação de CINE por critérios clínicos ou por positividade de provas funcionais. Foram avaliados o desempenho da TCMD para o diagnóstico de lesões > 50\% em segmentos coronários, artérias coronárias e pacientes e as estratégias de revascularização adotadas. Resultados: A sensibilidade, a especificidade e os valores preditivos positivo e negativo da TCMD foram de $86 \%, 95 \%, 71 \%$ e $100 \%$ para os segmentos coronários, de $91 \%, 92 \%, 80 \%$ e $100 \%$ para as artérias coronárias, e de $100 \%, 90 \%, 100 \%$ e $100 \%$ para os pacientes, respectivamente. No GP, a intervenção coronária percutânea (ICP) foi realizada em $90 \%$ dos pacientes, enquanto no GC a ICP foi realizada em apenas $43 \%(P=0,01)$. Conclusão: A TCMD demonstrou alto desempenho na detecção não-invasiva de DAC e proporcionou a realização de ICP ad hoc em 90\% dos pacientes. Essa estratégia, no entanto, deverá aguardar estudos randomizados que confirmem esses resultados.

DESCRITORES: Doença das coronárias. Angioplastia transluminal coronária percutânea. Angiografia coronária.

\footnotetext{
1 Hospital Beneficência Portuguesa de São Paulo - São Paulo, SP, Brasil.

2 Instituto de Cardiologia de São Paulo - São Paulo, SP, Brasil.

3 Hospital Evangélico Samaritano de Campinas - Campinas, SP, Brasil.

4 Centro Médico de Campinas - Campinas, SP, Brasil.

${ }^{5}$ Hospital do Rim da Universidade Federal do Estado de São Paulo/ Unifesp - São Paulo, SP, Brasil.

${ }^{6}$ Hospital São José - Hospital Beneficência Portuguesa de São Paulo - São Paulo, SP, Brasil.

Correspondência: Wilson Albino Pimentel Filho. Rua Maestro Cardim, 769 - Bela Vista - São Paulo, SP, Brasil - CEP 01323-900

E-mail: wilpm@uol.com.br

Recebido em: 7/9/2010 • Aceito em: 30/11/2010
}

\section{ABSTRACT}

\section{Evaluation of the Diagnostic Accuracy of Coronary Computed Tomographic Angiography}

Introduction: Coronary computed tomographic angiography (CCTA) has been widely accepted as a non-invasive diagnostic tool for coronary artery disease (CAD). The objective of this study was to evaluate the diagnostic performance of CCTA and its influence on modification of percutaneous revascularization strategies. Method: The study included two groups of patients: a main group (MG), including 100 patients screened with a suspect of severe CAD by CCTA and indication for coronary cineangiography (CINE), and a control group (CG) for comparison, including 100 patients selected during the same period, with indication for CINE according to clinical criteria or by positive functional tests. We evaluated the performance of CCTA for the diagnosis of lesions $>50 \%$ in coronary segments, arteries and patients and the revascularization strategies adopted. Results: The sensitivity, specificity and positive and negative predictive values of CCTA were $86 \%, 95 \%, 71 \%$ and $100 \%$ for the coronary segments, $91 \%, 92 \%, 80 \%$ and $100 \%$ for the coronary arteries and 100\%, 90\%, 100\% and $100 \%$ for patients, respectively. In the MG, percutaneous coronary intervention $(\mathrm{PCl}$ ) was performed in $90 \%$ of the patients, whereas in the CG, percutaneous coronary intervention was performed in $43 \%$ of the patients $(P=0.01)$. Conclusion: CCTA had a high diagnostic performance in detecting CAD and allowed ad hoc $\mathrm{PCl}$ to be performed in $90 \%$ of the patients. This strategy, however, must await randomized studies to confirm these results.

KEY-WORDS: Coronary disease. Angioplasty, transluminal, percutaneous coronary. Coronary angiography. 
A angiotomografia coronária de múltiplos detectores tem seu uso cada vez mais disseminado no meio cardiológico mundial, incluindo o brasileiro, como procedimento diagnóstico não-invasivo para detecção de doença arterial coronária. Esse exame avalia não somente a luz e o grau da obstrução arterial, como também as características da parede do vaso. ${ }^{1}$ Hoje os resultados dessa técnica apresentam excelente correlação com a cineangiocoronariografia, quando a lesão obstrutiva é > 50\%, com sensibilidade > 80\% e especificidade $>90 \%$. $^{2,3}$ Também é alto o valor preditivo negativo $(>90 \%)^{2,3}$, tornando-se um método confiável na exclusão de doença arterial coronária. O progresso da tecnologia desses aparelhos ao lado da experiência de seus operadores permite a apreciação integral das artérias coronárias, do perfil funcional de ambos os ventrículos e da perfusão do miocárdio. ${ }^{4-9}$

No entanto, é necessário reduzir a dose de radiação e a quantidade de contraste para que o método seja utilizado com maior segurança. Além disso, também tem sido debatido se a angiotomografia coronária de múltiplos detectores teria a precisão necessária para substituir a cineangiocoronariografia, discussão ainda sem resposta definitiva. ${ }^{10,11}$ Dentro dessa perspectiva, o objetivo deste estudo foi avaliar o desempenho diagnóstico da angiotomografia coronária de múltiplos detectores em nosso meio e sua influência na modificação das estratégias de revascularização percutânea.

\section{MÉTODO}

\section{Pacientes}

No período entre janeiro de 2007 e dezembro de 2009, triamos 100 pacientes com suspeita clínica de doença arterial coronária grave pela angiotomografia coronária de múltiplos detectores e indicação de cineangiocoronariografia, que compuseram o grupo principal (GP), os quais foram comparados a 100 pacientes selecionados de forma consecutiva no mesmo período, com indicação de cineangiocoronariografia por critérios clínicos ou por positividade de provas funcionais, compondo o grupo controle (GC). Os pacientes do GP foram encaminhados a cineangiocoronariografia/intervenção coronária percutânea por indicação de seu cardiologista e todos receberam terapia antiagregante dupla (aspirina 200 mg/dia e clopidogrel 750 mg/dia) pré-procedimento.

Foram excluídos do estudo pacientes com ritmo cardíaco irregular, com alergia ao contraste iodado, com insuficiência cardíaca congestiva, com creatinina $>1,5 \mathrm{mg} / \mathrm{dl}$ ou com contraindicação ao uso de betabloqueadores. O comitê de ética de todos os hospitais aprovou o protocolo e todos os pacientes assinaram o termo de consentimento.

\section{Procedimento de angiotomografia coronária de múltiplos detectores}

Inicialmente foi analisada a quantidade de cálcio arterial e obtido o escore de cálcio. Foram administra- dos por via oral, uma hora antes da obtenção das imagens, $100 \mathrm{mg}$ de atenolol (ou $50 \mathrm{mg}$ do mesmo fármaco caso a frequência cardíaca se situasse entre 50 bpm e 60 bpm). Durante o procedimento tomográfico, monitorizou-se a frequência cardíaca, a pressão arterial e o eletrocardiograma. Em alguns casos foi acrescentado metropolol (5 mg a $30 \mathrm{mg}$ ), por via intravenosa, para obter frequência cardíaca $<65$ bpm. Dinitrato de isossorbida sublingual na dose de $5 \mathrm{mg}$ foi administrado um minuto antes do início da aquisição das imagens tomográficas.

Utilizou-se a técnica helicoidal antes e durante a infusão endovenosa periférica de $110 \mathrm{ml}$ de contraste iodado, hidrossolúvel e não-iônico, com reconstruções multiplanares e tridimensionais das imagens obtidas com 64 detectores (Sensation 64 Cardiac, Siemens Medical Systems, Forchheim, Alemanha).

\section{Avaliação pela angiografia coronária convencional}

A cineangiocoronariografia foi realizada pela técnica de Judkins por punção femoral. A avaliação das lesões foi realizada em projeções ortogonais por dois ou mais observadores. O grau de obstrução dessas lesões foi então determinado pela angiografia coronária quantitativa (QuantCor, PI Medical Systems, Masstricht, Holanda).

\section{Comparação entre angiotomografia coronária de múltiplos detectores e angiografia coronária quantitativa}

O desempenho da angiotomografia coronária de múltiplos detectores para o diagnóstico de estenoses coronárias > 50\% foi comparado ao da angiografia coronária quantitativa, seguindo o protocolo: 1) análise por segmento (comparação de cada segmento de vaso); 2) análise por artéria (comparação de cada uma das principais artérias coronárias); e 3) análise por paciente. As estratégias de revascularização adotadas também foram avaliadas.

\section{Análise estatística}

A sensibilidade, a especificidade e os valores preditivos positivo e negativo da angiotomografia coronária de múltiplos detectores para a detecção de lesões > 50\%, reveladas pela angiografia coronária quantitativa, foram calculados por segmento, vaso e paciente. A medida de dependência estatística entre as mensurações realizadas pela angiotomografia coronária de múltiplos detectores e angiografia coronária quantitativa foi avaliada pelo coeficiente de correlação de Spearman ${ }^{12}$ e a concordância entre os dois métodos diagnósticos foi realizada pela análise de Bland-Altman. ${ }^{13}$ As variáveis contínuas foram apresentadas como média \pm desvio padrão e comparadas pelo teste $t$ de Student; as variáveis categóricas foram apresentadas como porcentuais e comparadas pelos testes de qui-quadrado ou exato de Fischer, quando apropriado. Em todos os testes, o valor de $\mathrm{P}<0,05$ foi considerado estatisticamente significante. 


\section{RESULTADOS}

Foram excluídos do GP 4 pacientes pela qualidade técnica inadequada da angiotomografia coronária de múltiplos detectores decorrente de arritmias detectadas antes do procedimento (fibrilação atrial, extrassístoles atriais ou ventriculares frequentes e disfunção do nó sinusal). Também foram excluídos 2 pacientes por alergia ao contraste e 1, por broncoespasmo. No GP, a frequência cardíaca basal durante a angiotomografia coronária de múltiplos detectores foi de $58 \pm$ 7,2 batimentos/minuto e $65 \%$ dos pacientes tinham escore de cálcio > 400 U Agatston.

As características clínicas e angiográficas dos pacientes tanto do GP como do GC estão sumarizadas na Tabela 1, e não apresentaram diferenças, à exceção da idade (61,3 \pm 10,4 anos vs. 66,3 \pm 12,7 anos, respectivamente; $\mathrm{P}<0,01)$ e do fato de que $23 \%$ de pacientes do GC não mostraram lesões coronárias significativas (> 50\%) à cinecoronariografia.

Foram analisados 935 de 1.065 segmentos coronários (88\%) de maneira qualitativa e quantitativa. Desses segmentos, 773 de 935 (83\%) foram mensurados quantitativamente pelas duas técnicas, angiotomografia coronária de múltiplos detectores e angiografia coronária quantitativa, e 355 dos 773 segmentos
$(45,9 \%)$ tinham lesões $>50 \%$. O desempenho da angiotomografia coronária de múltiplos detectores em termos de sensibilidade, especificidade e valores preditivos positivo e negativo para segmentos coronários, artérias coronárias e pacientes analisados encontra-se discriminado na Tabela 2.

O coeficiente de correlação de Spearman para o porcentual máximo de obstrução da luz entre as duas modalidades foi alto $(0,81 ; \mathrm{P}<0,001)$ (Figura 1). A análise de Bland-Altman demonstrou diferença média do grau de estenose de 1,3 $\pm 14,2 \%$, com intervalo de confiança de 95\% de 29,7\%-27,1\% (Figura 2).

As estratégias de revascularização adotadas também foram avaliadas. No GP, foi realizada intervenção coronária percutânea com sucesso em 90 pacientes (90\%), e 10 pacientes que não foram tratados percutâneamente: em 6 pacientes o grau da lesão era inferior ao visualizado na angiotomografia coronária de múltiplos detectores e em 4 pacientes a anatomia coronária não era apropriada ao procedimento, sendo encaminhados eletivamente a cirurgia de revascularização miocárdica. Nos multiarteriais, a intervenção coronária percutânea foi realizada em todos os vasos com lesões obstrutivas graves em 45 de 55 pacientes $(81,8 \%)$. O implante do stent farmacológico ocorreu em $38 \%$ dos pacientes.

TABELA 1

Características clínicas e angiográficas dos pacientes

\begin{tabular}{|c|c|c|c|}
\hline & $G P(n=100)$ & $\mathrm{GC}(n=100)$ & $\mathbf{P}$ \\
\hline Idade, média \pm desvio padrão & $61,3 \pm 10,4$ & $66,3 \pm 12,7$ & $<0,01$ \\
\hline Sexo masculino, \% & 64 & 59 & 0,56 \\
\hline Índice de massa corporal, kg/m² & $27,2 \pm 4,3$ & $28,3 \pm 5,4$ & 0,11 \\
\hline Tabagismo corrente, \% & 29 & 32 & 0,93 \\
\hline Hipertensão, \% & 49 & 46 & 0,77 \\
\hline Dislipidemia, \% & 67 & 65 & 0,88 \\
\hline Diabetes melito, \% & 33 & 30 & 0,76 \\
\hline Infarto prévio, \% & 31 & 29 & 0,88 \\
\hline Angioplastia prévia, \% & 22 & 20 & 0,86 \\
\hline Cirurgia prévia, \% & 10 & 8 & 0,81 \\
\hline Apresentação clínica, \% & & & 0,17 \\
\hline Angina estável & 56 & 43 & \\
\hline Instável & 24 & 33 & \\
\hline Outros & 20 & 24 & \\
\hline Creatinina, mg/dl & $0,97 \pm 0,2$ & $0,99 \pm 0,4$ & 0,66 \\
\hline Número de vasos com estenose $\geq 50 \%, \%$ & & & $<0,01$ \\
\hline 0 & 0 & 23 & \\
\hline 1 & 45 & 30 & \\
\hline 2 & 35 & 21 & \\
\hline 3 & 20 & 26 & \\
\hline
\end{tabular}

$\mathrm{GC}=$ grupo controle; GP = grupo principal. 
TABELA 2

Desempenho da angiotomografia coronária na detecção de lesões $>50 \%$ reveladas pela angiografia coronária quantitativa

\begin{tabular}{lcccc}
\hline & Sensibilidade & Especificidade & VPP & VPN \\
\hline Pacientes $(n=100), \%$ & 100 & 90 & 100 & 100 \\
Artérias $(n=230), \%$ & 91 & 92 & 80 & 100 \\
Segmentos $(n=880), \%$ & 86 & 95 & 71 & 100 \\
\hline
\end{tabular}

$\mathrm{n}=$ número; $\mathrm{VPN}=$ valor preditivo negativo; VPP = valor preditivo positivo.

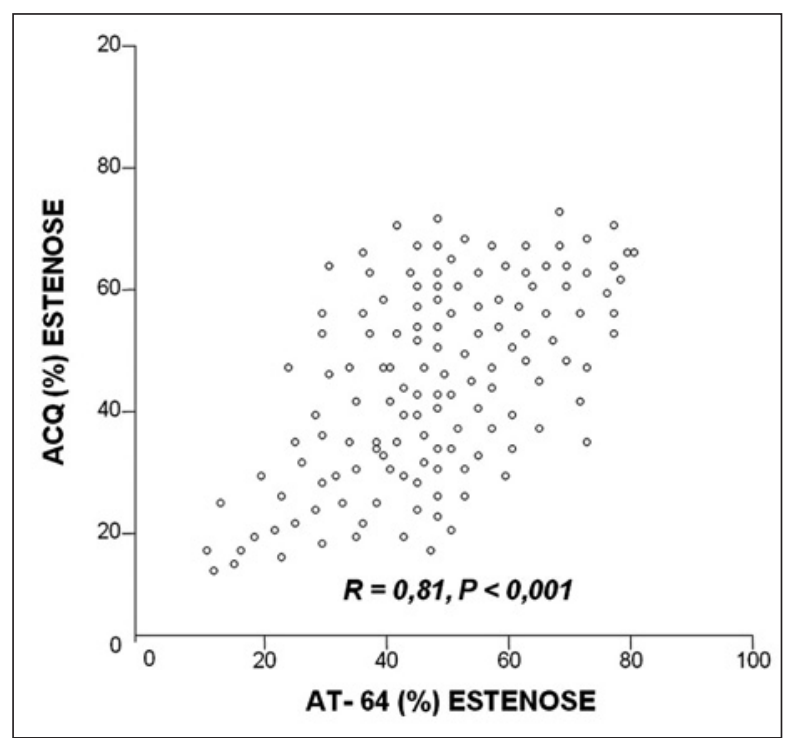

Figura 1 - Correlação do porcentual do diâmetro da estenose máximo entre angiotomografia coronária (AT-64) e angiografia coronária quantitativa (ACQ).

No GC a intervenção coronária percutânea foi realizada em $43 \%$ dos pacientes, comparativamente a $90 \%$ no GP $(P=0,01)$. Diferentemente do GP, no GC foi indicado tratamento clínico em $21 \%$, comparativamente a 0 no GP $(P=0,03)$, e cirurgia de revascularização miocárdica em $13 \%$, comparativamente a $4 \%$ no GP $(\mathrm{P}=0,04)$. O implante do stent farmacológico foi realizado em $36 \%$ dos pacientes.

\section{DISCUSSÃO}

Neste estudo, demonstramos que a angiotomografia coronária de múltiplos detectores apresentou bom desempenho diagnóstico para doença arterial coronária em comparação à angiografia coronária quantitativa. Além disso, contribuiu para "eliminar" uma etapa invasiva em 90\% dos pacientes submetidos a tratamento percutâneo. Por outro lado, seu alto poder em diagnosticar os segmentos que não apresentavam doença aterosclerótica (valor preditivo negativo) torna o método uma ferramenta não-invasiva respeitável para triar pacientes com fatores de risco para doença arterial coronária.

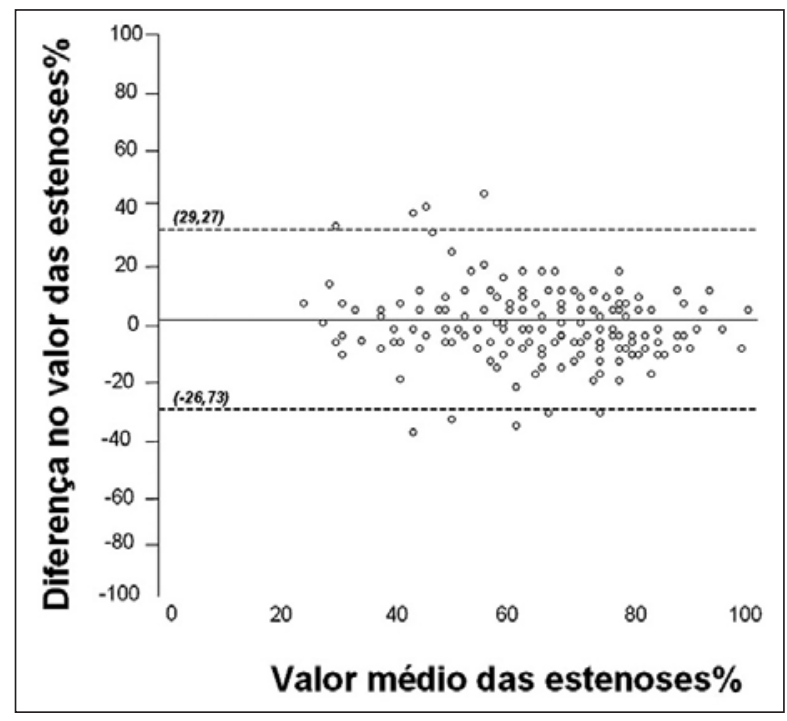

Figura 2 - Concordância entre angiografia coronária quantitativa e angiotomografia coronária pela análise de Bland-Altman para o porcentual do diâmetro de estenose da luz. A diferença média do grau de estenose foi de 1,3 $\pm 14,2 \%$ (linha central), com intervalo de confiança de $95 \%$ de $29,7 \%-27,1 \%$. Um total de $95 \%$ dos valores encontra-se entre as linhas paralelas, superior e inferior, com desvio padrão de 1,96.

Em relação à realização da intervenção coronária percutânea utilizando a angiotomografia coronária de múltiplos detectores como triagem, é importante ressaltar que a cineangiocoronariografia não foi eliminada, mas realizada durante o procedimento percutâneo, com avaliação angiográfica convencional completa, mas econômica (menor quantidade de contraste), de toda a árvore coronária. Assim, não eliminamos a cineangiocoronariografia, mas uma fase, o diagnóstico invasivo isolado. Após a confirmação angiográfica dos dados obtidos pela angiotomografia coronária de múltiplos detectores, foi realizado o tratamento percutâneo ad hoc em 90\% dos pacientes, com sucesso em 100\% deles. A falha da angiotomografia coronária de múltiplos detectores ao detectar casos favoráveis à intervenção coronária percutânea (grau de obstrução e/ou complexidade anatômica) em 10 pacientes indica que o método ainda não deve ser utilizado sem a comprovação angiográfica convencional completa da árvore coronária. 
Em nossa opinião, um aspecto relevante foi o fato de a pesquisa ter estudado as diferenças entre os grupos quanto à conduta para a realização de procedimentos angiográficos convencionais. Na amostra de pacientes "extremamente" selecionados pela angiotomografia coronária de múltiplos detectores no GP, demonstramos que foi possível realizar intervenção coronária percutânea na quase totalidade dos pacientes.

No entanto, temos que considerar que o aspecto que talvez tenha influenciado nossos resultados seja o fato de no GC as cineangiocoronariografias terem sido realizadas sem uma seleção de pacientes mais rigorosa por meio de testes funcionais complementares, reflexo da prática clínica em hospitais gerais. Pacientes com avaliação funcional pré-cineangiocoronariografia sugerindo doença arterial coronária grave tornariam esse grupo mais uniforme e mais próximo do GP, em termos de pacientes selecionados.

Apesar do desempenho atrativo da angiotomografia coronária de múltiplos detectores, ainda é preciso considerar alguns pontos importantes, como custo, exposição à radiação e quantidade relativamente alta de contraste para a realização desses procedimentos. ${ }^{14}$ É provável que, a curto prazo, esses dados negativos sejam minimizados com o aperfeiçoamento tecnológico. ${ }^{11,14-17}$

\section{Limitações do estudo}

Este estudo apresenta diversas limitações: 1) foi realizado de maneira retrospectiva e com a participação de apenas três centros; 2) pequena população de pacientes; e 3) indisponibilidade de informações relativas ao porcentual de pacientes submetidos a provas funcionais no GC ou a seu resultado.

Acreditamos que, apesar das referidas limitações, o estudo oferece a oportunidade de analisar e comparar os dados pertinentes a essa nova forma de avaliação não-invasiva da doença arterial coronária.

\section{CONCLUSÃO}

Em nosso estudo, a angiotomografia coronária de múltiplos detectores demonstrou alto desempenho na detecção não-invasiva de doença arterial coronária e proporcionou a realização de intervenção coronária percutânea ad hoc em 90\% dos pacientes. No entanto, não podemos estimular ou abalizar a adoção dessa estratégia até que estudos randomizados confirmem esses resultados.

\section{CONFLITO DE INTERESSES}

Os autores declararam inexistência de conflito de interesses relacionado a este manuscrito.

\section{REFERÊNCIAS}

1. Choi EK, Choi SI, Rivera JJ, Nasir K, Chang SA, Chun EJ, et al. Coronary computed tomography angiography as a screening tool for the detection of occult coronary artery disease in asymptomatic individuals. J Am Coll Cardiol. 2008;52(57): 357-65.

2. Raff GL, Gallagher MJ, O'Neill WW, Goldstein JA. Diagnostic accuracy of noninvasive coronary angiography using 64-slice spiral computed tomography. J Am Coll Cardiol. 2005;46(3): 552-7.

3. Schuijf JD, Pundziute G, Jukema JW, Lamb HJ, Salm LP, Van der Hoeven BL, et al. Diagnostic accuracy of 64-slice multislice computed tomography in the non-invasive evaluation of significant coronary artery disease. Am J Cardiol. 2006;98(2): 145-8.

4. Leschka S, Alkadhi H, Plass A, Desbiolles L, Grunenfelder J, Marincek B, et al. Accuracy of MSCT coronary angiography with 64-slice technology: first experience. Eur Heart J. 2005; 26(15):1482-7.

5. Cury R, Nieman K, Shapiro M, Nasir K, Cury RC, Brady T. Comprehensive cardiac CT study: evaluation of coronary arteries, left ventricular function, and myocardial perfusion. Is it possible? J Nucl Cardiol. 2007;14(2):229-43.

6. Fischbach $R$, Juergens $K U$, Ozgun $M$, Maintz D, Grude $M$, Seifarth $\mathrm{H}$, et al. Assessment of regional left ventricular function with multidetector-row computed tomography versus magnetic resonance imaging. Eur Radiol. 2007;17(4):1009-17.

7. Cury RC, Magalhães TA, Borges A, Parga J, Shiozaki A, Rochitte CE, et al. Dipyridamole stress and rest myocardial perfusion by 64 detector-row computed tomography in patients with suspected coronary artery disease. J Am Coll Cardiol. 2010;106(3):310-5.

8. Schepis T, Gaemperli O, Koepfli P, Valenta I, Strobel K, Brunner A, et al. Comparison of 64-slice CT with gated SPECT for evaluation of left ventricular function. J Nucl Med. 2006;47(8):1288-94.

9. Cury RC, Nieman K, Shapiro MD, Butler J, Nomura CH, Ferencik $\mathrm{M}$, et al. Comprehensive assessment of myocardial perfusion defects, regional wall motion, and left ventricular function by using 64-section multidetector CT. Radiology. 2008;248(2):466-75.

10. Van Werkhoven JM, Schuijf JD, Jukema JW, Pundziute G, de Roos A, Schalij MJ, et al. Comparison of non-invasive muti-slice computed tomography coronary angiography versus invasive coronary angiography and fractional flow reserve for the evaluation of men with know coronary artery disease. Am J Cardiol. 2009;104(5):653-6.

11. Dewey $M$, Zimmermann E, Deissenrieder F, Laule $M$, Dübel HP, Schlattmann P, et al. Noninvasive coronary angiography by 320-row computed tomography with lower radiation exposure and maintained diagnostic accuracy - comparison of results with cardiac catheterization in a head-to-head pilot investigation. Circulation. 2009;120(10):867-75.

12. Zimmerman DW, Williams RH. Properties of the Spearman correction for attenuation for normal and realistic non-normal distributions. Appl Psychol Meas. 1997;21(2):253-70.

13. Altman DG, Bland JM. Measurement in medicine: the analysis of method comparison studies. Statistician. 1983;32:307-17.

14. Ladapo JA, Jaffer FA, Hoffmann U, Thomson CC, Bamberg F, Cutler DM, et al. Clinical outcomes and cost-effectiveness of coronary computed tomography angiography in the evaluation of patients with chest pain. J Am Coll Cardiol. 2009; 54(25):2409-22.

15. Genders TSS, Meijboom WB, Meijs MFL, Schuijf JD, Mollet NR, Weustink AC, et al. CT coronary angiography in patients suspected of having coronary artery disease: decision making from various perspectives in the face of uncertainty. Radiology. 2009;253(3):734-44.

16. Graaf FR, Schuijf JD, van Velzen JE, Kroft LJ, Roos A, Reiber 
Pimentel Filho WA, et al. Avaliação da Acurácia Diagnóstica da Angiotomografia Coronária de Múltiplos Detectores. Rev Bras Cardiol Invasiva. 2010;18(4):429-34.

$\mathrm{JH}$, et al. Diagnostic accuracy of 320-row multidetector computed tomography coronary angiography in the noninvasive evaluation of significant coronary artery disease. Eur Heart J. 2010;31(15):1908-15.

17. Mark DB, Berman DS, Budoff MJ, Carr JJ, Gerber TC, Hecht
HS, et al. ACCF/ACR/AHA/NASCI/SAIP/SCAI/SCCT 2010 expert consensus document on coronary computed tomographic angiography: a report of the American College of Cardiology Foundation Task Force on Expert Consensus Documents. Catheter Cardiovasc Interv. 2010;76(2):E1-42. 